\title{
Herpes Zoster Vaccine: Time for a Boost?
}

\author{
Krishna P. Reddy, M.D. \\ Division of Pulmonary and Critical Care Medicine and Medical Practice Evaluation Center, Massachusetts General Hospital, Boston, \\ MA, USA.
}

$\mathrm{J}$ Gen Intern Med 32(2): 145-7

DOI: $10.1007 / \mathrm{s} 11606-016-3885-\mathrm{x}$

๑) Society of General Internal Medicine 2016

$\mathrm{O}$ ne in three persons in the US will develop herpes zoster (HZ) during their lifetime, and the risk increases with age. ${ }^{1}$ The disease is painful, often debilitating, and costly. Some cases result in postherpetic neuralgia (PHN), a chronic pain condition that can last months to years. In 2006, for the first time, a vaccine to prevent $\mathrm{HZ}$ and $\mathrm{PHN}$ became available in the US. A large-scale clinical trial in persons aged $\geq 60$ years had shown that the live attenuated vaccine reduced $\mathrm{HZ}$ incidence by $51 \%$ and PHN incidence by $67 \%$ over a median 3.1year follow-up period. ${ }^{2}$ Following this success, the Advisory Committee on Immunization Practices (ACIP) recommended one-time $\mathrm{HZ}$ vaccination for those $\geq 60$ years of age. ${ }^{1}$ However, a follow-up study of the original trial cohort reported a substantial decline in HZ vaccine efficacy over time; by year 11 post-vaccination, there was essentially no protection. ${ }^{3}$

If $\mathrm{HZ}$ vaccination is successful, and yet protection is shortlived, a key challenge is finding the "sweet spot" age at which to vaccinate. Such timing would protect people for the maximum length of time during which they are at highest risk of $\mathrm{HZ}$ and its complications. While the question of the optimal age at which to vaccinate remains, so does the related question of whether to administer a booster dose in the face of waning protection. Under the currently recommended strategy, those vaccinated only once at age 60 would be left unprotected after age 70, when the incidence and severity of HZ and PHN are highest. A recently published study reported fairly robust cellmediated immune responses after a booster dose in persons who had received an initial $\mathrm{HZ}$ vaccine 10 years earlier. ${ }^{4}$ The potential clinical benefit of a booster should be weighed against its added cost.

In this issue of the Journal of General Internal Medicine, Le and Rothberg report a methodologically robust and well-timed cost-effectiveness analysis which sought to determine the optimal schedule for the live attenuated HZ vaccine. ${ }^{5}$ This work builds upon their previously published and guidelinecited cost-effectiveness analysis of $\mathrm{HZ}$ vaccination. ${ }^{1,6}$ The authors updated their well-developed Markov model, newly incorporated data regarding the long-term efficacy of the vaccine, and evaluated strategies entailing booster doses. ${ }^{3,4}$

Published online October 11, 2016
They examined 11 strategies, including no vaccination, onetime vaccination at various ages, a booster dose after 10 years, and two booster doses 10 years apart. They included a comprehensive list of key input parameters, including HZ incidence and its associated complications and quality-of-life decrements, age- and time-dependent vaccine efficacy, adherence to boosters, and vaccine costs. Model-generated results per strategy included HZ and PHN cases, per-person qualityadjusted life-years (QALYs), and per-person total costs. The authors reported incremental cost-effectiveness ratios (ICERs) and used a commonly cited, albeit somewhat arbitrary (and often considered too low), US threshold where interventions with an ICER $<\$ 100,000 /$ QALY are considered cost-effective.

Among the 11 strategies evaluated, only four were deemed efficient and worthy of further consideration: no vaccination, one-time vaccination at age 70 , vaccination at age 70 followed by a booster 10 years later, and vaccination at age 60 followed by boosters 10 and 20 years later. Notably, the ACIP's currently recommended schedule, one-time vaccination at age 60 , was not among the efficient strategies. Vaccination at age 60 followed by two boosters provided the greatest clinical benefit, but its ICER was relatively high, at $\sim \$ 154,000 /$ QALY. Using costeffectiveness benchmarks alone, vaccination at age 70 followed by one booster was the optimal strategy, with an ICER of $\sim$ 37,000/QALY. Recognizing some uncertainty in their model parameters, Le and Rothberg varied their input values and demonstrated that their results were generally robust.

Could vaccination at age 60 followed by two boosters ever be cost-effective, given that it provided the greatest QALYs? Le and Rothberg found that this strategy became more attractive when there was higher vaccine efficacy $(>69 \%$ over 5 years), higher adherence to booster doses $(>67 \%)$, and lower vaccine price $(<\$ 156 /$ dose, compared with the base case price of $\$ 187.89 /$ dose). The ICER of this strategy generally exceeded $\$ 100,000 /$ QALY, meaning that it is unlikely to be cost-effective in most scenarios. However, in the US, interventions exceeding the $\$ 100,000 / \mathrm{QALY}$ cost-effectiveness threshold are frequently financed.

As with any model-based analysis, this study reflected a simplification of a complex disease and prevention process. In particular, clinical outcomes regarding $\mathrm{HZ}$ vaccine booster doses are unknown. Le and Rothberg assumed that the efficacy and waning rate of a booster dose would be the same as that of an initial dose. Recent data have suggested that a booster might induce greater immunity than an initial dose in persons $\geq 70$ years old. ${ }^{4}$ We anxiously await reports of the clinical short-term and 
long-term effectiveness of a booster dose to see whether it is similarly effective in preventing disease. If anything, Le and Rothberg may have underestimated the benefit of a single booster, and if it is greater than they assumed, strategies involving two boosters may become less relevant.

How can these results affect clinical and public health practice? There are three key questions: 1) Is the goal of vaccination to prevent $\mathrm{HZ}$, to prevent severe $\mathrm{HZ}$, or to prevent PHN? 2) Given waning vaccine efficacy, if a one-time vaccination strategy is to be employed, at what age should it occur? 3) Should a booster be administered? In Le and Rothberg's model, one-time vaccination at age 60 prevented more HZ cases, while one-time vaccination at age 70 prevented more PHN cases. Vaccination at age 70, compared with vaccination at age 60, was projected to provide greater per-person clinical benefits with lower per-person total costs, due to the higher incidence and severity of $\mathrm{HZ}$ and PHN among unprotected persons $\geq 70$ years of age compared with younger persons and the waning protection offered by a single vaccine dose. The model projected that adding a booster 10 years after initial vaccination was always more effective (at higher cost) than one-time vaccination at the initial age; this is not surprising. Taking these findings together, a reasonable approach, as the authors have suggested, would be to focus $\mathrm{HZ}$ vaccination efforts on those $\geq 70$ years of age - whether initial vaccination or booster dose - to prevent more cases of severe $\mathrm{HZ}$ and PHN.

The economic burden of $\mathrm{HZ}$ and PHN is substantial. The annual incidence of $\mathrm{HZ}$ is $\sim 1 \%$ in persons aged $\geq 60$ years; ${ }^{2}$ there are $>66$ million people in this age group in the US. Direct costs, which include those of outpatient visits, medications, emergency department visits, and hospitalizations, are approximately \$300-600 per acute HZ episode and \$700-900 per PHN case (\$2,200-5,400 for a longer-lasting PHN case). ${ }^{1,5}$ Costs per hospitalization, which occurs in 1-4\% of $\mathrm{HZ}$ episodes, are \$7,500-8,200. ${ }^{1,5}$ As the pain from $\mathrm{HZ}$ and PHN may be debilitating, indirect costs related to loss in productivity and leisure time can be considerable. In fact, PHN reduces quality of life by a magnitude similar to an acute disease such as Pneumocystis jiroveci pneumonia. ${ }^{7}$ To their credit, the ACIP's initial and updated recommendations on $\mathrm{HZ}$ vaccine use included discussions of costs and costeffectiveness analysis. ${ }^{1,8}$ A budget impact analysis, which would take into consideration the large number of people eligible for the $\mathrm{HZ}$ vaccine and the relatively high incidence of HZ and PHN, would be helpful in determining the population-level financial consequences of existing and alternative vaccine schedules.

A newer adjuvant $\mathrm{HZ}$ subunit vaccine ( $\mathrm{HZ} / \mathrm{su}$, two doses given 2 months apart), in a phase 3 trial in people $\geq 70$ years of age, had $90 \%$ efficacy in preventing $\mathrm{HZ}$ over a mean followup period of 3.7 years. ${ }^{9}$ This vaccine would certainly benefit those not eligible for vaccination with a live virus, and while these results are promising, the longer-term efficacy has yet to be reported. Similar to the live attenuated vaccine, real-life adherence to the two doses and vaccine cost will also influence the cost-effectiveness of $\mathrm{HZ} / \mathrm{su}$.

The ACIP's most recent guidelines on the live attenuated HZ vaccine, published in 2014, did not change their initial recommendation of one-time vaccination in adults $\geq 60$ years of age, despite citing an internal costeffectiveness analysis that demonstrated a preference for vaccination at age $70 .^{8}$ Noting the methodological challenges of determining long-term $\mathrm{HZ}$ vaccine efficacy (the long-term follow-up study used a statistical model to estimate disease incidence in an unvaccinated historical control group ${ }^{3}$ ), the guidelines state: "Because duration of protection offered by the vaccine is uncertain, the need for revaccination is not clear." 8 In addition to follow-up trial data, recent Kaiser Permanente cohort data have added to the evidence for declining effectiveness (to an estimated $4 \%$ by year 8 ) after one-time $\mathrm{HZ}$ vaccination, ${ }^{10}$ so it may be time to revisit this notion in the guidelines. A booster might even be warranted sooner than 10 years after initial vaccination. The ACIP's currently recommended $\mathrm{HZ}$ vaccine strategy is likely not providing sufficient protection for those who need it most. While we await further studies of the durability of-and adherence to-boosters, Le and Rothberg's analysis suggests that we reconsider the optimal timing of initial dosing and booster dosing.

Acknowledgments: The author thanks Dr. Rochelle P. Walensky for her critical review of the manuscript.

Corresponding Author: Krishna P. Reddy, M.D.; Division of Pulmonary and Critical Care Medicine and Medical Practice Evaluation Center, Massachusetts General Hospital, 50 Staniford Street, 9th Floor, Boston, MA 02114, USA (e-mail: kpreddy@mgh.harvard.edu).

\section{Compliance with Ethical Standards:}

Conflict of Interest: The author reports no conflict of interest.

\section{REFERENCES}

1. Harpaz R, Ortega-Sanchez IR, Seward JF, Advisory Committee on Immunization Practices (ACIP) Centers for Disease Control and Prevention (CDC). Prevention of herpes zoster: recommendations of the Advisory Committee on Immunization Practices (ACIP). MMWR Morb Mortal Wkly Rep. 2008;57:1-30.

2. Oxman MN, Levin MJ, Johnson GR, et al. A vaccine to prevent herpes zoster and postherpetic neuralgia in older adults. N Engl J Med. 2005;352:2271-84.

3. Morrison VA, Johnson GR, Schmader KE, et al. Long-term persistence of zoster vaccine efficacy. Clin Infect Dis. 2015;60:900-9.

4. Levin MJ, Schmader KE, Pang L, et al. Cellular and humoral responses to a second dose of herpes zoster vaccine administered 10 years after the first dose among older adults. J Infect Dis. 2016;213:14-22. 
5. Le $\mathbf{P}$, Rothberg $\mathbf{M}$. Determining the optimal vaccination schedule for herpes zoster: a cost-effectiveness analysis. J Gen Intern Med. doi:10. 1007/s11606-016-3844-6

6. Rothberg MB, Virapongse A, Smith KJ. Cost-effectiveness of a vaccine to prevent herpes zoster and postherpetic neuralgia in older adults. Clin Infect Dis. 2007;44:1280-8.

7. Freedberg KA, Scharfstein JA, Seage GR, et al. The costeffectiveness of preventing AIDS-related opportunistic infections. JAMA. 1998;279:130-6.
8. Hales CM, Harpaz R, Ortega-Sanchez I, Bialek SR, Centers for Disease Control and Prevention (CDC). Update on recommendations for use of herpes zoster vaccine. MMWR Morb Mortal Wkly Rep. 2014;63:729-731.

9. Cunningham AL, Lal H, Kovac M, et al. Efficacy of the herpes zoster subunit vaccine in adults 70 years of age or older. N Engl $\mathrm{J}$ Med. 2016;375:1019-32.

10. Tseng HF, Harpaz R, Luo Y, et al. Declining effectiveness of herpes zoster vaccine in adults aged $\geq 60$ years. J Infect Dis. 2016;213:1872-5. 